\title{
Preliminary assessment of patients' opinions of queuing for coronary artery bypass graft surgery at one Canadian centre
}

\author{
Jean F Petrie, Jafna L Cox, Robert J Teskey, Lisa B Campbell, David E Johnstone
}

Division of Cardiology, Victoria General Hospital

Jean F Petrie, nursing manager, coronary care unit

Jafna L Cox, staff cardiologist

Robert J Teskeystaff, cardiologist

David E Johnstone, professor of medicine, head of division

Division of Cardiovascular Surgery, Victoria General Hospital Lisa B Campbell, divisional secretary

Department of Community Health and Epidemiology, Dalhousie University, Halifax, Nova Scotia, Canada

Jafna L Cox

Correspondence to: Dr David E Johnstone Division of Cardiology, Victoria General Hospital, 1278 Tower Road, Halifax Nova Scotia, Canada, B3H 2 Y9

Accepted for publication 13 June 1996

\begin{abstract}
Objectives-To explore psychological and socioeconomic concerns of patients who queued for coronary artery bypass surgery and the effectiveness of support existing in one Canadian cardiovascular surgical centre.

Design-Standardised questionnaire and structured interview.

Setting-Victoria General Hospital, Halifax, Nova Scotia.

Subjects-100 consecutive patients awaiting non-emergency bypass surgery.

Results-Most patients (96\%) found the explanation of findings at cardiac catheterisation and the justification given for surgery satisfactory. However, 84 patients complained that waiting for surgery was stressful and 64 registered at least moderate anxiety. Anger over delays was expressed by $16 \%$, but only $4 \%$ thought that queuing according to medical need was unfair. Economic hardship, attributed to delayed surgery, was declared by 15 patients. This primarily affected those still working - namely, blue collar workers and younger age groups. Only $41 \%$ of patients were satisfied with existing institutional supports. Problems related mainly to poor communication.

Conclusions-Considerable anxiety seems to be experienced by most patients awaiting bypass surgery. Better communication and education might alleviate some of this anxiety. Economic hardship affects certain patient subgroups more than others and may need to be weighed in the selection process. A more definitive examination of these issues is warranted.
\end{abstract}

\section{Introduction}

Lengthy delays for elective medical services in Canada reflect the tension between the promise of universal care and finite medical resources. ${ }^{1}$ Waiting for coronary artery bypass graft surgery has, in particular, generated intense criticism. In the late $1980 \mathrm{~s}$, a dramatic increase in referrals for bypass surgery overtook caseload growth. ${ }^{2}$ Nationally, the wait for elective operations approached 23 weeks ${ }^{3}$ and some patients died while waiting. A more efficient and appropriate triage process was proposed ${ }^{4}$ and widely adopted, with regional modifications. Preliminary data suggested that the vital risk associated with queuing was small, ${ }^{5-7}$ and the ethics of such managed delay has been defended. ${ }^{8}$ Yet few studies have assessed the effect of waiting on general health related quality of life issues, ${ }^{910}$ and there has been little insight from the perspective of patients.

Questions of increased morbidity or mortality aside, delayed surgery has potentially profound psychological and socioeconomic implications which may reflect more accurately the true burden of the queue. ${ }^{11}$ These include persistent symptoms, anxiety, and costs to both the person and society. A prospective assessment of the process used to triage patients for bypass surgery at our cardiovascular surgical centre $^{7}$ provided an opportunity to launch a substudy aimed at acquiring a preliminary understanding of patient satisfaction with queuing, to explore some related psychosocial stresses, and to examine the adequacy of existing support for patients.

\section{Methods}

PATIENT TRIAGE PROCESS

The Victoria General, a teaching hospital of Dalhousie University, provides all cardiovascular surgery to the provinces of Nova Scotia and Prince Edward Island, a population of about one million people. Six cardiovascular surgeons perform about 1000 open heart operations each year.

The process for prioritising patients for surgery is strictly controlled and shares features common with similar systems elsewhere in Canada. ${ }^{4}$ Uniquely, however, all patients referred for consideration of revascularisation are reviewed and discussed at a weekly conference of cardiovascular specialists. They are then stratified by consensus to a four tiered urgency ranking scheme. The symptoms, anatomy, and functional status of referred patients are the only issues considered. Urgent patients, who are critically ill, generally undergo surgery within a week of presentation at conference and are kept in hospital until their operative date. Two semiurgent categories are recognised. Semiurgent A patients, with cardiac instability or life threatening anatomy, ideally undergo surgery within two to four weeks. Patients ranked as semiurgent $B$, with advanced symptoms and unable to exercise for more than five metabolic equivalents (METS) according to the Bruce protocol despite maximum medical treatment, have a target of four to 10 weeks wait. Finally, elective patients, who may be doing poorly on medical treatment but 
nevertheless are capable of exercising more than five METS, have a target of 10-16 weeks waiting time.

STUDY POPULATION

The absence of data on patient satisfaction with waiting for bypass surgery in Canada led us to undertake a descriptive exploratory study to acquire preliminary insights. A separate evaluation of our triage process, already planned, involved prospective follow up of all patients referred for isolated bypass surgery at our institution from 1 April 1992 to 31 October 1992. Detailed demographic and clinical data, including patient employment status, were collected on 423 patients and have been reported. ${ }^{7}$ From this parent population, 100 consecutive patients awaiting surgery on a non-emergency basis were approached for the present substudy. On providing informed consent, they received a standardised questionnaire administered by an interviewer.

QUESTIONNAIRE

A simple four part questionnaire was drafted. The areas selected related to programme quality control issues or were thought to be of concern to patients. As an add on to a more involved study, and because we sought to maximise patient participation, the number of questions was deliberately limited for easy and rapid use. The intention was not to develop a comprehensive instrument for assessing patient satisfaction, which is a complex task, ${ }^{12-17}$ but rather to acquire background data for such an undertaking in the future. The first section examined whether findings at cardiac catheterisation had been adequately communicated, and whether both the justification for surgery and the prioritisation process had been effectively explained.

The second section related to the amount of anxiety attributable to the surgical delay. Patients were asked to rate their anxiety level as none, mild, moderate, or severe. Also, they were asked whether they were angered by the waiting process and, if so, whether this related to the delay itself or to a perception that prioritisation based on medical need was unfair.

A third section inquired about patient satisfaction with the support received from the surgical centre. We ascertained whether dissatisfaction related to lack of ongoing communication and follow up, lack of a clear booking date, or to some other issue which patients were asked to specify. All patients undergoing bypass surgery on a nonemergency basis at the Victoria General Hospital are sent an educational package com-

Table 1 Distribution of surgical priority rankings of patients by age groups

\begin{tabular}{|c|c|c|c|c|c|c|}
\hline \multirow[b]{2}{*}{$\begin{array}{l}\text { Surgical priority } \\
\text { rankings }\end{array}$} & \multicolumn{5}{|c|}{ Age groups (y) } & \multirow[b]{2}{*}{ Total } \\
\hline & $<50$ & $50-59$ & $60-69$ & $70-79$ & $\geqslant 80$ & \\
\hline Urgent & 1 & 2 & 5 & 8 & 0 & 16 \\
\hline Semiurgent A & 1 & 2 & 1 & 3 & 1 & 8 \\
\hline Semiurgent B & 7 & 11 & 14 & 9 & 2 & 43 \\
\hline Elective & 6 & 12 & 12 & 3 & 0 & 33 \\
\hline Total & 15 & 27 & 32 & 23 & 3 & 100 \\
\hline
\end{tabular}

$\mathrm{P}=0.139$. prising pamphlets which describe in lay terms the atherosclerotic process, surgical revascularisation, and the anticipated perioperative course. We asked whether this package had been received and, if so, whether it had been helpful.

In the final section, patients were asked whether delayed surgery imposed economic hardship. At each stage, additional comments were solicited and recorded.

The questionnaire was given to all patients by one interviewer, a coronary care unit head nurse with over 25 years of experience (JFP). Because this person was on a research sabbatical, and thus was not associated with the clinical care of any of the patients, it was anticipated that respondents would be more forthright with her in terms of expressing distress or dissatisfaction than if interviewed by an active member of the healthcare team. Interviews were held in private during the surgical admission. Scripted questions were read in a standard order. When subjective interpretations, such as mild, moderate, or severe, were requested, their definition was left up to the patients. All patients were encouraged to provide additional comments at each stage and these were recorded verbatim.

DATA ACQUISITION, ENTRY, AND ANALYSIS

Results were entered as categorical variables into a computer spreadsheet program. Analysis was by $\chi^{2}$ or, when cell counts were low, Fisher's exact test. Patients' comments were grouped into broad categories for the purpose of uncovering subjective insights but were not subjected to statistical analysis.

\section{Results}

PATIENT CHARACTERISTICS

All of 100 consecutive patients admitted for non-emergency bypass surgery and approached to participate in our satisfaction substudy consented to do so. They were interviewed at a median of one day before operation (range one to two). This cohort was $72 \%$ men and $28 \%$ women. The mean age was 62 (range 37-84). By comparison, the triage study population of 423 patients, from which our subgroup was drawn, was $72 \%$ men and $28 \%$ women with a mean age of 64 (range 31-88).

Priority rankings were 16 urgent, eight semiurgent A, 43 semiurgent $B$, and 33 elective. Comparable ratings for the parent population were $35 \%$ urgent, $9.7 \%$ semiurgent $\mathrm{A}, 39 \%$ semiurgent $B$, and $16.3 \%$ elective. Thus fewer urgent but more elective patients, who waited longest and were most likely to be affected by long delays, were sampled. No age bias was evident in the assignment of priority rankings (table 1).

Only 31 patients continued to work outside the home, 14 in blue collar (manual work) and 17 in white collar (predominantly office based) positions. Table 2 shows patient employment status stratified by surgical priority ranking. Most patients who remained at home were retired, although one fifth $(15 / 69,22 \%)$ stated 
Table 2 Employment status of patients stratified by surgical priority ranking

\begin{tabular}{|c|c|c|c|c|}
\hline \multirow[b]{2}{*}{$\begin{array}{l}\text { Surgical priority } \\
\text { rankings }\end{array}$} & \multicolumn{3}{|c|}{ Employment status } & \multirow[b]{2}{*}{ Total } \\
\hline & $\begin{array}{l}\text { Not } \\
\text { working }\end{array}$ & $\begin{array}{l}\text { Working } \\
\text { employee }\end{array}$ & $\begin{array}{l}\text { Outside } \\
\text { home self } \\
\text { employed }\end{array}$ & \\
\hline Urgent & 14 & 2 & 0 & 16 \\
\hline Semiurgent A & 7 & 1 & 0 & 8 \\
\hline Semiurgent B & 28 & 12 & 3 & 43 \\
\hline Elective & 20 & 9 & 4 & 33 \\
\hline Total & 69 & 24 & 7 & 100 \\
\hline
\end{tabular}

$\mathrm{P}=0.392$. If all patients working are compared with all patients not working, $\mathrm{P}=0.207$.

that they were disabled by their cardiac condition and hence unable to work.

\section{QUESTIONNAIRE RESPONSES}

Most patients (96\%) thought that both the explanation of findings at cardiac catheterisation and the justification given for surgery had been satisfactory. Waiting generated some anxiety in $84 \%$ but was moderate for $55 \%$ and severe for $9 \%$. The burden of anxiety was similar across priority rankings (table 3 ) but was slightly greater in the younger age groups (table 4$)$. Thus $76 \%(32 / 42)$ of patients under 60 had at least moderate anxiety versus 55\% $(32 / 58)$ of those 60 or older. Anger over waiting time was expressed by 16 patients, of whom two experienced mild, 10 moderate, and four severe anxiety. Angry patients were uniformly upset by having to wait at all for major surgery. However, when the issue of equity was raised, given that limited resources meant that invariably some waiting was required, only four patients thought that prioritisation on the basis of medical need was unfair. Two of these were ranked urgent and hence were operated upon promptly. The other two, one ranked semiurgent $B$ and the other elective, were also angered by the duration of their surgical delays, which they perceived to be excessive.

Economic hardship caused by delayed surgery was declared by 15 patients. Those still working $(10 / 31,32 \%)$ were affected more than those not working for whatever reason $(5 / 69$, $7 \%, \mathrm{P}=0.002)$. Workers in blue collar occupations $(8 / 14,57 \%)$ were more affected than their white collar counterparts $(2 / 17,12 \%$, $\mathrm{P}=0.018$ ). Younger patients also had disproportionately more economic hardship (table 5).

Only $41 \%$ of patients were totally satisfied with support from the institution. Poor communication was cited as a major problem by 47 patients. Several complained about delays which were longer than anticipated, and

Table 3 Patient anxiety level and anger by surgical priority ranking

\begin{tabular}{|c|c|c|c|c|c|c|c|}
\hline \multirow[b]{2}{*}{$\begin{array}{l}\text { Surgical priority } \\
\text { rankings }\end{array}$} & \multicolumn{4}{|c|}{ Anxiety level } & \multicolumn{3}{|l|}{ Anger } \\
\hline & None & Mild & Moderate & Severe & Waiting & Unfair & Both \\
\hline Urgent & 0 & 4 & 12 & 0 & 3 & 2 & 0 \\
\hline Semiurgent A & 3 & 0 & 3 & 2 & 1 & 0 & 0 \\
\hline Semiurgent B & 6 & 9 & 23 & 5 & 7 & 1 & 1 \\
\hline Elective & 7 & 7 & 17 & 2 & 5 & 1 & 1 \\
\hline Total & 16 & 20 & 55 & 9 & 16 & 4 & 2 \\
\hline
\end{tabular}

$\mathrm{P}=0.980$ for anxiety level; $\mathrm{P}=0.980$ for anger with waiting times; $\mathrm{P}=0.291$ for anger over perceived unfairness.
Table 4 Level of patient anxiety and anger stratified by age groups

\begin{tabular}{lllllllll}
\hline & \multicolumn{3}{l}{ Anxiety level } & & \multicolumn{2}{l}{ Anger } \\
\cline { 2 - 4 } \cline { 6 - 7 } Age $(y)$ & None & Mild & Moderate & Severe & & \multicolumn{2}{l}{ Waiting Unfair } & Both \\
\hline$<50$ & 1 & 3 & 7 & 4 & 3 & 0 & 0 \\
5059 & 6 & 0 & 20 & 1 & & 3 & 2 \\
$60-69$ & 5 & 10 & 14 & 3 & & 3 & 1 & 0 \\
$\geq 70$ & 4 & 7 & 14 & 1 & & 3 & 0 & 0 \\
Total & 16 & 20 & 55 & 9 & & 16 & 4 & 2 \\
\hline
\end{tabular}

$\mathrm{P}=0.020$ for anxiety level; $\mathrm{P}=0.149$ for anger with waiting

times; $P=0.310$ for anger over perceived unfairness.

Table 5 Perceived economic hardship by age

\begin{tabular}{llll}
\hline & \multicolumn{2}{l}{ Economic hardship } & P value \\
\cline { 2 - 3 } Age $(y)$ & Yes & No & \\
\hline$<50$ & 7 & 8 & \\
$50-59$ & 6 & 21 & \\
$60-69$ & 2 & 30 & $<0.0001$ \\
$\geq 70$ & 0 & 26 &
\end{tabular}

in some instances longer than those projected for their urgency rankings. Many patients were not aware that waiting times were calculated from the date of the surgical conference rather than from catheterisation, a time difference of as much as two or three weeks. Others thought that the priority rankings assigned were less urgent than they had been led to expect. Unclear booking dates were an issue for 29 patients. Routine procedure involved quoting patients an approximate waiting time according to their assigned priority ranking; two days before the date booked for their surgical admission, they were contacted by telephone and asked to come to hospital. Patients wanted more time to plan their schedules and prepare emotionally for surgery.

Only $55 \%$ of patients received an education package before their surgical admission. A further $32 \%$ received the package on admission, but $13 \%$ did not receive it at all. The information was considered helpful by most patients who received their package $(72 / 87$, $83 \%$ ) and was significantly more beneficial if received before the surgical admission (54/55, $98 \%)$ than after $(18 / 32,56 \%, P<0.0001)$.

\section{Discussion}

Waits for medical services are ubiquitous in Canada, and in other countries where health care is publicly funded. It is therefore surprising that relatively few studies have examined the impact of queuing from the perspective of patients. Most studies have been concerned with issues of safety and fairness. Our report highlights additional psychological and socioeconomic concerns.

Very high levels of patient satisfaction (> $85 \%$ ) with their care in general, ${ }^{17}$ and with their physicians in particular, ${ }^{18}$ have been reported. One recent Canadian study ${ }^{18}$ found that only $7 \%$ to $16 \%$ of patients reported problems with the way education on medications or tests was provided. Our patients overwhelmingly (96\%) thought that both the findings at cardiac catheterisation and the rationale for surgery had been adequately communicated to them. However, the prioritisation process was 
less clearly understood and several further problems developed once the wait for surgery began.

Delayed surgery gave at least moderate anxiety in $64 \%$, was greatest for younger patients, but interestingly was experienced equally across all surgical priority rankings. Patients ranked as urgent, who went for surgery promptly at a mean of 2.8 days, $^{7}$ were as anxious as those ranked elective, who waited as long as six months. This suggests that something other than waiting time was contributing to anxiety. Possibilities include fear of open heart surgery itself, or lack of understanding of the palliative nature of most bypass surgery, and the fact that waiting, despite ongoing symptoms, is associated with little vital risk. ${ }^{819}$ Improved patient education and reassurance might reduce the anxiety.

Overall, economic hardship was uncommon. This may reflect in part that many patients requiring bypass surgery are elderly and retired. Some of these would have been financially secure. Others would depend on private or public pension payments. By contrast, younger patients would more likely be carrying debts and many would be primary or even sole providers for their families. For them, protracted symptoms might result in temporary or permanent loss of employment. Sick benefits or compensation payments might not redress sufficiently the lost income. Consistent with these hypotheses was that economic hardship was clearly concentrated among the employed and younger age groups. As economic hardship may impose an inequitable burden on some people, then perhaps it should be included in the selection process.

Dissatisfaction with support from the institution was common and generally resulted from poor communication. Lack of understanding of the prioritisation process led to uncertainty about operative dates, and educational material was often received too late for benefit. Steps have already been taken to redress these deficiencies. Educational material is now provided at the time surgery is first discussed or when notifying patients of their priority ranking. The recent establishment of a telephone hotline, to deal with patients' questions and concerns, aims to reduce any sense of isolation occurring from surgical delays.

This study has several limitations. Firstly, the number of patients interviewed was small and their experience was that of a single surgical centre. Nevertheless, to the extent that the Victoria General Hospital is the sole provider of bypass surgery for the Provinces of Nova Scotia and Prince Edward Island, the views of the people surveyed should be representative at least of the population of patients requiring cardiac revascularisation from these provinces. We suspect that patients faced with similar medical systems elsewhere would raise similar concerns.

Secondly, the time frame of the study was brief. However, neither the nature of bypass surgery nor the reality of surgical waits, and hence their impact on patients, have changed substantially over the past decade. Indeed, we think that the concerns raised by our patients on the anxiety of waiting, the socioeconomic burden of the queue, and the need for effective communication and education are specific to the experience of waiting for major surgery rather than to the era of the interviews.

Thirdly, we realised that questioning patients on the eve of their operation risked confounding the anxiety relating to their wait with that of the imminent major surgery. The net effect could have been to increase anxiety, due to additional apprehension about the surgical outcome, or to decrease it, given relief that the waiting had effectively ended. We nevertheless chose the timing to sample the extreme of each patient's waiting period, thereby comparing what we thought to be the most consistent and appropriate interval. Multiple interviews conducted throughout the waiting period may have provided insights into whether and how patients' views and anxiety changed over time. However, these would have been logistically difficult to arrange. More importantly, because waiting times are variable, especially for the least urgent priority rankings, intervals between interviews would have varied widely between patients thereby complicating substantially our ability to compare and interpret the results. Whatever the impact of the timing of the interview on anxiety and expressed levels of distress and dissatisfaction, anxiety is clearly considerable in most patients. Because some patients may bear a disproportionate burden of anxiety, should this be considered in the triage process? Unfortunately, we know of no adequately validated tools for objectively assessing relative anxiety between cardiac patients. Without such an instrument, patients could feign anxiety in an attempt to undergo earlier surgery thereby moving ahead of cases with more urgent medical needs. The development of such a measure should be a research priority.

Fourthly, because of our small sample size, we examined only a few issues. We suspect that this list is far from exhaustive and recognise that further details would require a much larger study. As well as being limited in scope, our questionnaire was also simple in design, as a comprehensive study of patient preferences and satisfaction was not planned at the time. Instead, as a necessary preliminary to the development of a more definitive and methodologically robust questionnaire, we simply sought to survey key areas of suspected patient and provider interest.

Despite these limitations, we would argue that our data do provide insights into several concerns that patients have with queuing for bypass surgery and we think that they permit some general observations and preliminary recommendations. Most importantly, healthcare providers need to be aware that considerable anxiety is experienced by most patients waiting for bypass surgery and economic hardship disproportionately affects certain groups. Imparting clear information to patients on their condition and the consequent need for 


\section{Appendix: coronary artery bypass graft questionnaire}

Thank you for taking time today to participate in our study. We are interested in improving our service to our patients. First, we need to understand better how you felt about the way you were managed.

We will ask you a series of questions. If there is anything you do not understand, then please feel free to ask questions. As stated in the consent form, participation in this study will not affect your medical care. You will not be identified, so you need not be concerned about offending anyone. Furthermore, you are free to stop the interview at any time.

Do you have any questions?

Let's begin:

1a After you had your cardiac catheterisation (the dye test), your doctor discussed the results with you and advised you to have surgery. Were the results of the test and the reasons for needing surgery adequately explained?

1b Are there any comments which you wish to make about the discussion?

(Record verbatim)

2a Has the time spent waiting for surgery caused you any anxiety?

(If No then skip to $2 c$, otherwise continue)

$2 \mathrm{~b}$ Would you say that your level of anxiety was "mild", "moderate" or "severe"?

2c Did having to wait for bypass surgery make you angry?

2d Patients who need emergency surgery have their operation immediately. All others must wait. However, the length one waits depends on medical need. Do you believe that a policy of having the sickest patients go first is fair?

2e Are there any comments which you wish to make regarding your wait for surgery?

(Record verbatim)

3a Did waiting for surgery cause you any economic hardship or financial difficulty?

$3 \mathrm{~b}$ Are there any comments which you wish to make about how waiting for surgery may have affected your economic or financial situation?

(Record verbatim)

4a Once the decision to proceed with surgery was made, were you totally satisfied with the support you received?

(If Yes then skip to $4 \mathrm{e}$; if satisfaction was anything less than total, continue)

$4 \mathrm{~b}$ Was there a problem with ongoing communication?

$4 \mathrm{c}$ Was there a problem with a clear booking date?

$4 \mathrm{~d}$ Did you encounter any other problem, or problems?

(Record verbatim)

$4 \mathrm{e}$ Did you receive an education package containing information about your operation

before this admission?

4f Did you find the information helpful?

(Record verbatim)

That's the end of the interview. Again, thank you very much for your time.

surgery is important. Preferably, this should involve clear and ongoing discussion with the healthcare team as well as easily understood printed educational materials delivered in a timely fashion. It is essential that such communications enable patients to understand why their surgery is being delayed, how priority rankings are assigned, and the low risks associated with waiting. It is noteworthy that $96 \%$ of patients who queued thought that prioritisation based on medical need was fair. Presumably, most patients are willing to tolerate delays as the cost of universal access to health services. In return, physicians must minimise as far as possible risk to patients, discomfort, and anxiety, and must speak up when resources are so restricted that queues jeopardise patients or make their distress intolerable. Finally, current triage systems for bypass surgery establish priority according to medical need, specifically relative to symptom level or the anatomical nature of the disease. But if a disproportionate amount of anxiety and economic burden is indeed borne by younger patients and by people in particular employment settings, as our findings suggest, then an important equity issue is raised namely, whether certain socioeconomic variables should be considered explicitly when triaging patients. This matter warrants further study.

JLC is supported by Medical Research Council and Pharmaceutical Manufacturers Association of Canada Development Grant PA-12825.

1 Naylor CD. The Canadian health care system: a model for America to emulate? Health Economics 1992;1:19-37.

2 Ugnat A-M, Naylor CD. Trends in coronary artery bypass grafting in Ontario from 1981 to 1989 . Can Med Assoc $\mathcal{F}$
. grafting in Ontario

3 Higginson LAJ, Cairns JA, Keon WJ, Smith ER. Rates of cardiac catheterisation, coronary angioplasty and openheart surgery in adults in Canada. Can Med Assoc 7 1992; 146:921-5.

4 Naylor CD, Baigrie RS, Goldman RS, Basinski A. Revascularization Panel and Consensus Methods Group: assessment of priority for coronary revascularization procedures. Lancet 1990;335:1070-3.

5 Carrier M, Pineault R, Tremblay N, Pelletier C. Outcome of rationing access to open-heart surgery: effect of the wait for elective surgery on patient outcome. Can Med Assoc 7 1993;149:1117-22.

6 Naylor CD, Morgan CD, Levinton CM, Wheeler S, Hunter $\mathrm{L}, \mathrm{Klymciw} \mathrm{K}$, et al. Waiting for coronary revascularization in Toronto: two years in Toronto: two years experience with a regional referra

7 Cox JL Petrie JF, Pollak PT, J

for coronary artery bypass delay one Canadian center. $\Im$ Am Coll Cardiol 1996;27:1365-73. 
8 Cox JL. Ethics of queuing for coronary artery bypass grafting in Canada. Can Med Assoc F 1994;151:949-53.

9 Mulgan R, Logan RL. The coronary bypass waiting list: a social evaluation. $N Z$ Med f 1990;103:371-2.

10 Pieper B, Lepezyk M, Caldwell M. Perceptions of the waiting period before coronary artery bypass grafting. Heart Lung 1985;14:40-4.

11 Naylor CD. A different view of queues in Ontario. Health Affairs (Millwood) 1991;10:110-28.

12 Del Greco L, Walop W. Questionnaire development: 1. Formulation. Can Med Assoc ₹ 1987;136:583-5.

13 mulation. Can Med Assoc $f_{\text {W. Marthy RH }}$ development: 2. Validity and reliability. Can Med Assoc $\mathcal{F}$ 1987:136:699-700.

14 Health Services Research Group. Studying patients' preferences in health care decision making. Can Med Assoc J 1992;147:859-64.
15 Froberg DG, Kane RL. Methodology for measuring healthstate preferences - I: measurement strategies. $₹$ Clin Epidemiol 1989;42:345-54.

16 Froberg DG, Kane RL. Methodology for measuring healthstate preferences - II: scaling methods. $f$ Clin Epidemio 1989;42:459-71.

17 Carr-Hill RA. The measurement of patient satisfaction. $\mathcal{f}$ Public Health Med 1992;14:236-49.

18 Charles C, Gauld M, Chambers L, O'Brien B, Haynes RB. How was your hospital stay? Patients' reports about their care in Canadian hospitals. Can Med Assoc $\mathcal{F} 1994$ 150:1813-22.

19 Cox J, Naylor CD. The Canadian Cardiovascular Society grading scale for angina of effort: Is it time for refinements? Ann Intern Med 1992;117:677-83. 